\title{
Two-Color RESOLFT Nanoscopy with Green and Red Fluorescent Photochromic Proteins**
}

\author{
Flavie Lavoie-Cardinal, ${ }^{[a]}$ Nickels A. Jensen, ${ }^{[a]}$ Volker Westphal, ${ }^{[a]}$ Andre C. Stiel, ${ }^{[a]}$ \\ Andriy Chmyrov, ${ }^{[a]}$ Jakob Bierwagen, ${ }^{[a]}$ Ilaria Testa, ${ }^{[a]}$ Stefan Jakobs, ${ }^{*[a, b]}$ and Stefan W. Hell ${ }^{*[a]}$
}

\begin{abstract}
Up to now, all demonstrations of reversible saturable optical fluorescence transitions (RESOLFT) superresolution microscopy of living cells have relied on the use of reversibly switchable fluorescent proteins (RSFP) emitting in the green spectral range. Here we show RESOLFT imaging with rsCherryRev1.4, a new red-emitting RSFP enabling a spatial resolution up to
\end{abstract}

four times higher than the diffraction barrier. By co-expressing green and red RSFPs in living cells we demonstrate two-color RESOLFT imaging both for single ("donut") beam scanning and for parallelized versions of RESOLFT nanoscopy where an array of $>23000$ "donut-like" minima are scanned simultaneously.

\section{Introduction}

Throughout the 20th century the resolution of far-field optical microscopy has been limited by diffraction to about $250 \mathrm{~nm}$, a value given by half the wavelength $\lambda$ of light divided by the numerical aperture (NA) of the objective lens. ${ }^{[1]}$ At the turn of this century, the diffraction barrier has been radically overcome $^{[2]}$ and since then several far-field fluorescence nanoscopy or superresolution methods have been developed. ${ }^{[3]}$ At the end of the day, all these methods exploit a transition between two fluorophore states, usually an "on" (fluorescent) and an "off" (dark) state in order to discriminate adjacent features. ${ }^{[3 b]}$ Depending on the way they implement the two-state transition for feature separation, two major classes of fluorescence nanoscopy can be identified, namely coordinate-stochastic and coordinate-targeted approaches. ${ }^{[3]}$ The stochastic approaches, such as those called photoactivated localization microscopy

[a] Dr. F. Lavoie-Cardinal, ${ }^{+}$Dr. N. A. Jensen, ${ }^{+}$Dr. V. Westphal, Dr. A. C. Stiel, Dr. A. Chmyrov, Dr. J. Bierwagen, Dr. I. Testa, Prof. Dr. S. Jakobs,

Prof. Dr. S. W. Hell

Department of NanoBiophotonics

Max Planck Institute for Biophysical Chemistry

37070 Göttingen (Germany)

Fax: (+ 49) 551 201-2505

E-mail: shell@gwdg.de

[b] Prof. Dr. S. Jakobs

Department of Neurology

University Medical Center of Göttingen

37073 Göttingen (Germany)

E-mail:sjakobs@gwdg.de

$\left.{ }^{+}\right]$Equal contributions

[**] RESOLFT = reversible saturable optical fluorescence transitions

Supporting Information for this article is available on the WWW under http://dx.doi.org/10.1002/cphc.201301016.

of $\odot 2014$ The Authors. Published by Wiley-VCH Verlag GmbH\&Co. KGaA This is an open access article under the terms of the Creative Commons Attribution-NonCommercial-NoDerivs License, which permits use and distribution in any medium, provided the original work is properly cited, the use is non-commercial and no modifications or adaptations are made.
$(\mathrm{PALM})^{[4]} /$ stochastic optical reconstruction microscopy (STORM), ${ }^{[5]}$ ground-state depletion with individual-molecule return $(\mathrm{GSDIM})^{[6]} /$ direct STORM (dSTORM), ${ }^{[7]}$ single-molecule active-control microscopy $(\mathrm{SMACM}){ }^{[8]}$ and others, induce this transition randomly in space to create individualized on-state fluorophores; the fluorophores surrounding them remain in the off-state. On the other hand, the coordinate-targeted methods, ${ }^{[2]}$ such as stimulated emission depletion (STED) microscopy, ${ }^{[2 b, 9]}$ saturated patterned excitation microscopy $(\mathrm{SPEM}) /$ saturated structured illumination microscopy $(\mathrm{SSIM})^{[10]}$ or reversible saturable optical fluorescence transitions (RESOLFT) ${ }^{[2,11]}$ induce the state transition with a light intensity pattern having local minima at predefined positions in the focal plane. The position of the minimum (ideally zero) and the maximum intensities of the light pattern jointly determine the coordinates where the fluorophores are allowed to assume a specific state; usually it is the on-state.

Typically detecting several fluorophores simultaneously, coordinate-targeted modes such as STED and RESOLFT microscopy have the potential to image very fast, allowing the visualization of dynamic processes in living cells or even tissue. ${ }^{[12]}$ This is particularly true for STED microscopy, which uses the very fast process of stimulated emission from the fluorescent singlet state, $S_{1}$, to the singlet ground state, $S_{0}$, as the feature-separating on-off state transition. However, ensuring a nearly constant occupation of the $S_{0}$ calls for relatively large stimulating light intensities, because of the relatively short (nanosecond) lifetime of the $S_{1}$ state. Since the related RESOLFT nanoscopy concept typically uses metastable on- and off-states of reversibly switchable fluorophores, ${ }^{[2,, 11 a, b]}$ the light intensities needed to create the required difference in states are several orders of magnitude lower than in STED microscopy. Therefore, RESOLFT nanoscopy can be implemented with light intensity patterns having well over ten thousand minima (zeros) so that large fields of view can be recorded within relatively short times. ${ }^{[2]}$ In fact, a parallelized RESOLFT approach using two incoherently 
superimposed orthogonal standing light waves has recently been used to image $100-150 \mu \mathrm{m}$ sized fields of view in a few seconds. ${ }^{[13]}$ Moreover, the much lower light intensities needed for RESOLFT nanoscopy, as compared to all other nanoscopy methods, underscore the suitability of the RESOLFT concept for extended imaging of living cells and tissues. ${ }^{[12 a, 14]}$

In RESOLFT nanoscopy relying on reversibly switchable fluorescent proteins (RSFPs), the spectral properties, the switching kinetics, and the photostability of the RSFPs used are vitally important. Clearly, the RSFPs need also to provide many sequential on- and off-cycles and a high contrast between the on- and the off-states. The recent development and exploration of novel RSFPs such as rsEGFP1, ${ }^{[11 c]}$ rsEGFP2, ${ }^{[12 a]}$ Dronpa M159T $^{[14,15]}$ and Dreiklang ${ }^{[11 d]}$ has enabled RESOLFT nanoscopy in living cells. Yet, all RSFPs used so far for RESOLFT live-cell imaging emit in the yellow-green spectral regime, peaking at 500-530 nm. This hampered two-channel RESOLFT recordings, making colocalisation studies by using two spectrally distinct RSFPs impossible.

Interestingly, as a matter of fact, in the initial demonstration of the RESOLFT concept, ${ }^{[11 a]}$ a red-emitting RSFP called asFP595 was used. However, presumably like the fluorescent proteins used in early switching demonstrations, ${ }^{[16]}$ asFP595 does not fulfill the criteria for implementing RESOLFT imaging. Concretely, asFP595 forms tetramers, and exhibits poor photostability, making biological imaging very difficult. While monomeric red emitting RSFPs such as rsCherryRev, rsCherry ${ }^{[17]}$ and rsTagRFP ${ }^{[18]}$ were developed in the succeeding years, RESOLFT imaging has not been demonstrated with these proteins. Here, we report on the development of a two-color RESOLFT nanoscopy scheme using red and green fluorescent RSFPs, and introduce the newly developed RSFP rsCherryRev1.4 for use in singlebeam point-scanning and highly parallelized versions of RESOLFT nanoscopy.

\section{Results and Discussion}

\section{1. rsCherryRev1.4, an Improved Red Fluorescent Reversible Switchable Protein for RESOLFT Nanoscopy}

The previously developed RSFP rsCherry $\operatorname{Rev}^{[17]}$ has high photostability, high on-off contrast and low switching fatigue, but at the same time low overall folding efficiency, rendering it less suitable for demanding imaging applications such as RESOLFT nanoscopy. Therefore, we decided to further improve the properties of rsCherryRev to make it suitable for RESOLFT. After several rounds of mutagenesis and screening we identified the rsCherryRev variant rsCherryRev1.4 (rsCherryRev-G29C, R41H, R130C, V149F, R154Q, E165L) (Figure S1, Table S1, Supporting Information). The numbering in the name of this new variant is meant to indicate that this protein is likely to be further optimized; its limitations are detailed in the following.

The RSFP rsCherryRev1.4 exhibits similar spectral characteristics as its predecessor rsCherryRev. In thermal equilibrium it is in the non-fluorescent off-state that exhibits an absorption maximum at $572 \mathrm{~nm}$ (Figure $1 \mathrm{a}$ ). The fact that the thermal equilibrium is the off-state renders a precise determination of a

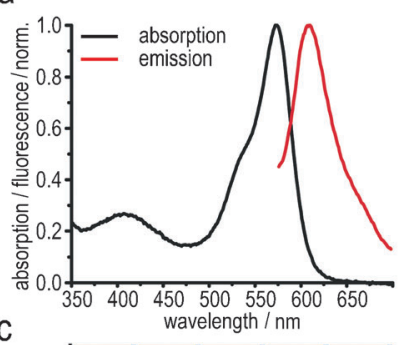

C

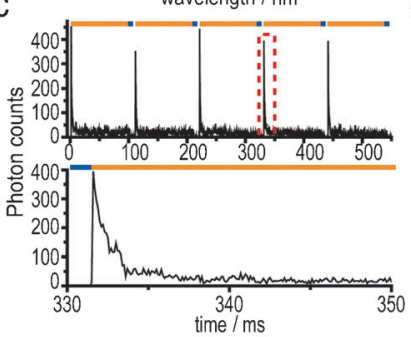

b
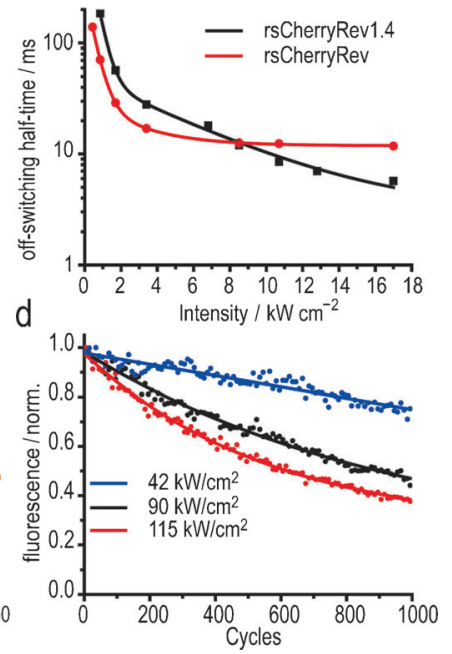

Figure 1. Protein characteristics. a) Normalized absorption spectrum of the non-fluorescent equilibrium state (black) and fluorescence emission spectrum (red) of rsCherryRev1.4. Spectra were recorded at pH 7.5. For the emission spectrum, the proteins were initially switched into the on-state. b) Intensity dependence of the off-switching half-time for rsCherryRev1.4 (black) and rsCherryRev (red) measured on living HeLa cells expressing the respective vimentin-fusion proteins, duration of exposure was $1 \mathrm{~s}$. At intensities $>10 \mathrm{~kW} \mathrm{~cm}^{-2}$, rsCherryRev1.4 shows faster off-switching kinetics than rsCherryRev. c) Top: Several switching cycles of rsCherryRev1.4 in living cells. On-switching was performed with $430 \mathrm{~nm}$ at $0.5 \mathrm{~kW} \mathrm{~cm}^{-2}$ for $0.5 \mathrm{~ms}$ and offswitching was performed with $592 \mathrm{~nm}$ at $115 \mathrm{~kW} \mathrm{~cm}^{-2}$ for $100 \mathrm{~ms}$. Bottom: Magnification of the region marked in the upper graph, demonstrating that for this intensity the full switching-off process is accomplished in less than $5 \mathrm{~ms}$. d) Bleaching curves of rsCherryRev1.4 with different off-switching intensities measured on vimentin-rsCherryRev1.4 filaments. The on-switching parameters were kept constant $\left(430 \mathrm{~nm} ; 0.5 \mathrm{~kW} \mathrm{~cm}^{-2} ; 0.5 \mathrm{~ms}\right)$ and the offswitching step was performed in $10 \mathrm{~ms}$ with $592 \mathrm{~nm}$ laser intensity of $42 \mathrm{~kW} \mathrm{~cm}^{-2}$ (blue), $90 \mathrm{~kW} \mathrm{~cm}^{-2}$ (black) and $115 \mathrm{~kW} \mathrm{~cm}^{-2}$ (red). The fluorescence was recorded for 1000 cycles, averaged for 10 consecutive cycles, and normalized to the maximal intensity.

the on-state extinction coefficient and quantum yield challenging. The fluorescence emission maximum of the on-state is at $609 \mathrm{~nm}$. At $\mathrm{pH}$ values $<5.0$, rsCherryRev1.4 shows an additional absorption peak at $450 \mathrm{~nm}$ corresponding to the neutral state of the chromophore. The $\mathrm{p} K_{\mathrm{a}}$ of the off-state chromophore is 5.5 (Figure S2). Both rsCherryRev1.4 and rsCherryRev exhibit negative switching characteristics, that is, irradiation with light of $\sim 570 \mathrm{~nm}$ induces fluorescence and, in a competing process, switches the protein off, while irradiation at $\sim 430 \mathrm{~nm}$ converts the protein back to the on-state. To investigate the switching behavior under RESOLFT imaging conditions, the coding sequence of rsCherryRev1.4 was fused to the coding sequence of vimentin and the fusion construct was expressed in cultivated human HeLa cells.

The switching properties were analyzed for light intensities typically used for RESOLFT imaging $\left(1-100 \mathrm{~kW} \mathrm{~cm}^{-2}\right)$. For switching, light of $430 \mathrm{~nm}$ (on-switching) and $592 \mathrm{~nm}$ (offswitching and fluorescence read-out) was used. As previously reported for rsCherryRev, the time required for off-switching is about two orders of magnitude longer than for on-switching at comparable laser intensity, rendering the off-switching time as the speed-limiting step within a switching cycle. At compa- 
ratively low light intensities $\left(<8 \mathrm{~kW} \mathrm{~cm}^{-2}\right)$, the off-switching of rsCherryRev was equivalent or faster compared to rsCherryRev1.4. However, at higher light intensities $\left(>10 \mathrm{~kW} \mathrm{~cm}^{-2}\right)$, offswitching of rsCherryRev1.4 was faster, while the off-switching speed of rsCherryRev could not be further reduced (Figure $1 \mathrm{~b}$ ). Because off-switching is the time-limiting step in RESOLFT nanoscopy with negative switching RSFPs, the shorter off-switching time of rsCherryRev1.4 represents an important advantage over rsCherryRev for live-cell RESOLFT applications. Using $592 \mathrm{~nm}$ light of intensity $>30 \mathrm{~kW} \mathrm{~cm}^{-2}$, the ensemble fluorescence signal was reversibly reduced to $<10 \%$ of the equilibrium value within $10 \mathrm{~ms}$ (Figure 1c).

To investigate the switching fatigue of rsCherryRev1.4, we measured the overall fluorescence decrease over 1000 full switching cycles of vimentinrsCherryRev1.4 expressed in living cells. The on-switching parameters and off-switching times were kept constant, but different intensities were employed for the off-switching. We found that even at high off-switching light intensities of $115 \mathrm{~kW} / \mathrm{cm}^{2}$, the protein could undergo more than 600 switching cycles in living cells before the fluorescence was reduced to $50 \%$. At $42 \mathrm{~kW} \mathrm{~cm}^{-2}$, the overall fluorescence was reduced by only $20 \%$ after 1000 cycles (Figure $1 \mathrm{~d}$ ).

Native gel electrophoresis showed that rsCherryRev1.4 tends to dimerize (Figure S2 d). Yet, various rsCherryRev1.4 fusion proteins including fusions with keratin 19, vimentin, $\alpha$-tubulin, caveolin and MAP2 could be expressed in cultivated human HeLa cells; they all exhibited the correct sub-cellular localization (Figure S3). This presumably indicates that the dimerization tendency in living cells is weaker than that indicated by gel electrophoresis, although this tendency may represent a severe hurdle in case of other host proteins. Targeted to the endoplasmic reticulum or several other cellular structures, the overall cellular brightness observed in case of cells expressing rsCherryRev1.4 fusion proteins was on average about twice as high as in the case of cells expressing the corresponding rsCherryRev fusion protein (Figure $\mathrm{S} 3 \mathrm{f}, \mathrm{g}$ ). This may indicate a higher folding efficiency of rsCherryRev1.4, although the chromophore maturation times of both proteins were similar $\left(t_{1 / 2} \sim 40 \mathrm{~min}\right)$ (Figure S2c). In brief, rsCherryRev1.4 exhibits low switching fatigue enabling more than 1000 switching cycles at light intensities of $40 \mathrm{~kW} \mathrm{~cm}^{-2}$ facilitating an off-switching time of only $\sim 10 \mathrm{~ms}$. Although it tends to dimerize, rsCherryRev1.4 can be used to tag several cellular host proteins.

\subsection{Single-Beam Scanning RESOLFT Nanoscopy with Red Fluorescent RSFPs}

To investigate whether rsCherryRev1.4 can be used for RESOLFT microscopy, we built a single-beam RESOLFT microscope for red fluorescent RSFPs. The setup was implemented as a stage-scanning confocal microscope equipped with three different lasers. The length and the delay for each irradiation step were controlled with nanosecond precision using acousto-optical devices (Figure S4). Imaging was performed by scanning the sample stage. At every pixel, rsCherryRev1.4 was first switched into the on-state with a regularly focused beam of $430 \mathrm{~nm}\left(0.5-1 \mathrm{~kW} \mathrm{~cm}^{-2}\right)$ for $0.1-0.5 \mathrm{~ms}$. Then, the fluorophores at the rim of the focal spot of excitation light were switched 
into the off-state by a donut-shaped beam of $592 \mathrm{~nm}$ $\left(20 \mathrm{~kW} \mathrm{~cm}^{-2}-50 \mathrm{~kW} \mathrm{~cm}^{-2}\right)$ for 3-10 ms. Finally the fluorescence was read out with a focused beam of $561 \mathrm{~nm}\left(1-5 \mathrm{~kW} \mathrm{~cm}^{-2}\right)$ for 0.1-1 ms (Figure S5). Contrary to previous RESOLFT implementations using green RSFPs, ${ }^{[11 c, 12 a, 14]}$ here the off-switching and reading-out were performed at different wavelengths. rsCherryRev1.4 fluorescence is most efficiently excited at $561 \mathrm{~nm}$. However, by switching off at this wavelength, the ensemble brightness could not be reduced below $17 \%$, possibly because of action cross-talk with the on-switching process (Figure S6). Therefore, we decided to switch the protein off with light of $592 \mathrm{~nm}$ wavelength, reducing the residual fluorescence to $<7 \%$ of the maximum level. We found that upon increasing the fluorescence probing time to up to $1 \mathrm{~ms}$ at low light intensities $\left(<10 \mathrm{~kW} \mathrm{~cm}^{-2}\right)$, the signal-to-noise ratio (SNR) was markedly increased without noticeably compromising the resolution (Figure S7). Therefore, the probing time was adapted to the respective image brightness.

For RESOLFT microscopy, rsCherryRev1.4 was fused to the coding sequences of vimentin, keratin19, caveolin or the microtubule associated protein MAP2, expressed in HeLa cells and imaged with the irradiation sequence outlined above (Figure 2). The actual switching times and light intensities were adapted to the brightness and to the mobility of the respective cellular structures (Table S2). To record the largely immobile filaments formed by vimentin-rsCherryRev1.4 or keratin19rsCherryRev1.4, longer pixel dwell times of 10-12 ms and low light intensities (off-switching $<40 \mathrm{~kW} \mathrm{~cm}^{-2}$ ) were chosen, thus also maximizing the resolution and reducing phototoxicity. We found caveolin-rsCherryRev1.4 and rsCherryRev1.4-MAP2 to exhibit faster movements, hence a shorter pixel dwell time was implemented. By increasing the laser intensity applied, the offswitching step was kept as short as $3 \mathrm{~ms}$. For all structures imaged, the resolution increase was clearly visible, meaning that details that were blurred in the confocal images could be discerned in their RESOLFT counterparts (Figure 2).

The obtained resolution was assessed by measuring the diameter of filaments formed by keratin19-rsCherryRev1.4 in living HeLa cells. To this end, we averaged five neighboring intensity profiles across thin filaments within $100 \mathrm{~nm}$, and fitted the profiles with a Lorentzian function. Altogether thirteen filaments were analyzed. On average we measured a filament diameter of $58 \pm 7 \mathrm{~nm}$; the diameters of the smallest filaments were $<50 \mathrm{~nm}$ (Figure $2 \mathrm{~b}$ and Figure $\mathrm{S} 7 \mathrm{a}, \mathrm{b}$ ). As the measured filament diameter is co-determined by the actual size of the filaments, we conclude that the focal plane resolution was better than $50 \mathrm{~nm}$ in the living cell.

To find out whether rsCherryRev1.4 can be used for timelapse live-cell investigations, cells expressing rsCherryRev1.4MAP2 were continuously imaged for $13.5 \mathrm{~min}$, taking one image frame of size $3.5 \mu \mathrm{m} \times 3.5 \mu \mathrm{m}$ within $\sim 75$ seconds (Figure 3). Even after 10 frames, the recorded fluorescence signal was still $>90 \%$ of its initial value, demonstrating the good photostability of rsCherryRev1.4 and the potential for extended live cell imaging using this protein. The RESOLFT images show movements of rsCherryRev1.4-MAP2 decorated microtubules which are blurred in the confocal counterparts.

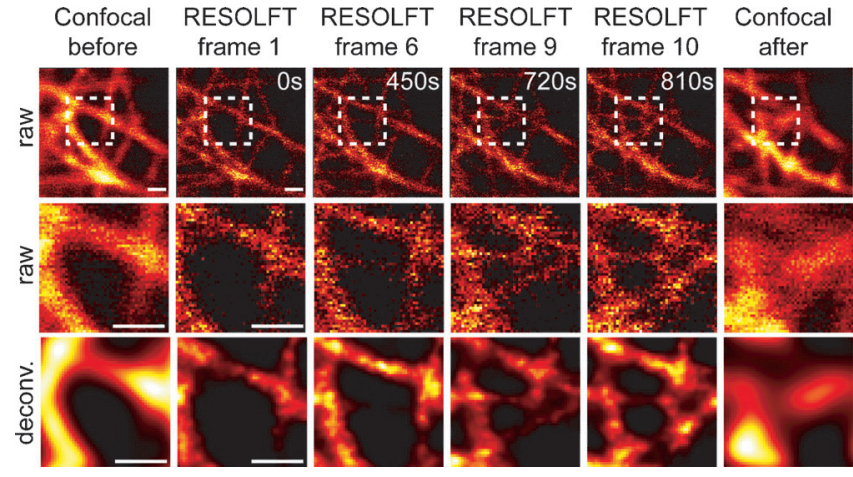

Figure 3. Continuous time-lapse RESOLFT imaging. Confocal and RESOLFT images of living HeLa cells expressing rsCherryRev1.4-MAP2. The confocal images were taken before and after the RESOLFT time lapse series. The time-lapse images show the formation of new filaments that can be distinguished in the RESOLFT image only. Note that the fluorescence intensity decreases by only $10 \%$ within 10 frames. Top: $3.5 \times 3.5 \mu \mathrm{m}^{2}$ field of view (raw data). Middle: Magnification of the indicated area (raw data). Bottom: Richardson-Lucy deconvolution of the same magnified area. Pixel step size: $25 \mathrm{~nm}$. Pixel dwell time: $3.8 \mathrm{~ms}$ (on: $0.3 \mathrm{~ms}, 0.8 \mathrm{~kW} \mathrm{~cm}^{-2}$; off: $3 \mathrm{~ms}$, $113 \mathrm{~kW} \mathrm{~cm}^{-22}$; read-out: $0.5 \mathrm{~ms}, 8 \mathrm{~kW} \mathrm{~cm}^{-2}$ ). Scale bars: $500 \mathrm{~nm}$.

Altogether, live-cell RESOLFT nanoscopy is viable with the red fluorescent protein rsCherryRev1.4 at an optical resolution comparable to that previously obtained with RSFPs emitting in the green range.

\subsection{Two-Color Single-Beam RESOLFT Nanoscopy}

Because the protein rsCherryRev1.4 is spectrally different from the green fluorescent RSFPs rsEGFP/rsEGFP2 ${ }^{[11 c, 12 a]}$ or Dronpa-

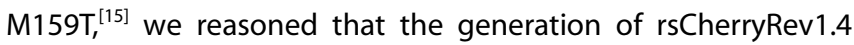
could facilitate two-color live-cell RESOLFT nanoscopy. rsCherryRev1.4 as well as rsEGFP and Dronpa-M159T exhibit negative switching behavior. Besides, the wavelengths inducing the offstate is longer by about $100 \mathrm{~nm}$ for the red as compared to the green RSFPs $(\sim 590 \mathrm{~nm}$ vs. $\sim 490 \mathrm{~nm})$, and all RSFPs can be conveniently switched on at $430 \mathrm{~nm}$.

Hence we designed a sequence of switching events that was expected to facilitate two-color RESOLFT microscopy using four laser lines $(430 \mathrm{~nm}, 488 \mathrm{~nm}, 561 \mathrm{~nm}$ and $592 \mathrm{~nm})$ (Figure 4). According to this scheme, rsEGFP2 and rsCherryRev1.4 are first transferred to the on-state by irradiation at $430 \mathrm{~nm}$ wavelength. Subsequently, rsCherryRev1.4 is switched to the off-state by a donut-shaped beam at $592 \mathrm{~nm}$ leaving only the proteins in the donut center in the on-state. The onstate rsCherryRev1.4 proteins are probed by a focused beam of $561 \mathrm{~nm}$, a wavelength at which rsEGFP2 is not excited. To subsequently image rsEGFP2, a donut-shaped beam at $488 \mathrm{~nm}$ is used for the off-switching, followed by a final probing beam at $488 \mathrm{~nm}$ wavelength. After this cycle, the sample is moved and the irradiation scheme is repeated until the entire sample has been scanned.

This irradiation scheme was implemented in the singlebeam point-scanning RESOLFT microscope by adding a donutshaped beam at $488 \mathrm{~nm}$ for switching rsEGFP2 off, and a regularly focused one for reading it out. The green fluorescence 


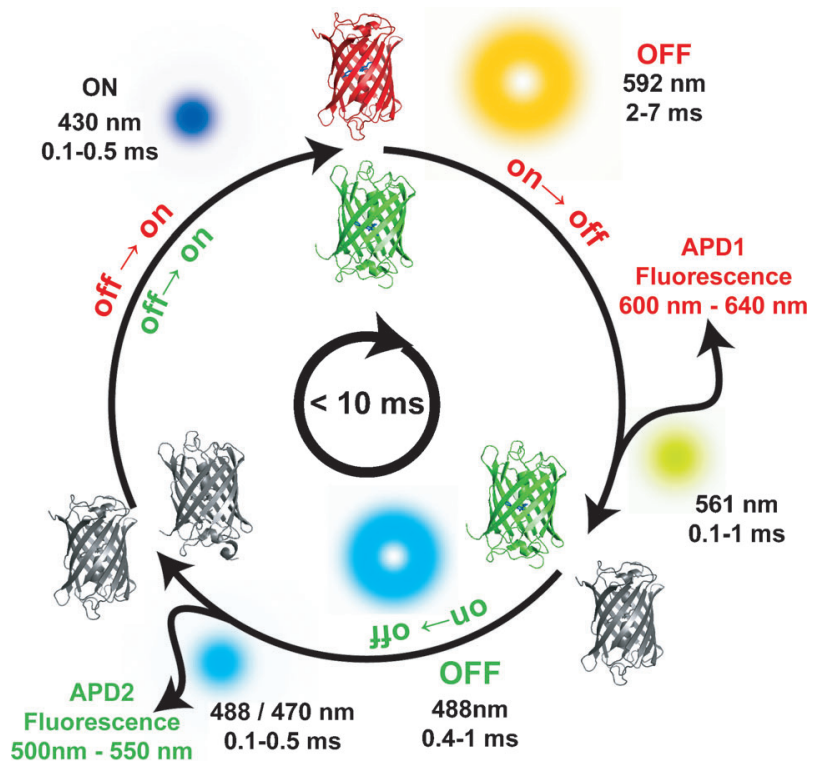

Figure 4. Switching scheme for two-color RESOLFT imaging. At first, the red and green fluorescent RSFPs are switched to their fluorescent on-state with a focused beam of $430 \mathrm{~nm}$ wavelength. The red proteins at the rim of the excitation focal spot are then switched to their off-state with a donutshaped beam of $592 \mathrm{~nm}$. The remaining molecules in the on-state are excited with a normally focused beam of $561 \mathrm{~nm}$ wavelength and the resulting red fluorescence is detected between 600 and $640 \mathrm{~nm}$. Afterwards, the green proteins are switched to their off-state with a donut shaped beam of $488 \mathrm{~nm}$; subsequently the green fluorescence is read out with a regularly focused beam of $488 \mathrm{~nm}$ wavelength. Depending on the laser intensity used, the time needed for a full switching cycle is between 6 and $10 \mathrm{~ms}$.

was detected on a second detector between $500 \mathrm{~nm}$ and $550 \mathrm{~nm}$. A custom-made dichroic mirror reflecting between $380-500 \mathrm{~nm}$ and $550-600 \mathrm{~nm}$ and transmitting between 500$550 \mathrm{~nm}$ and $600-660 \mathrm{~nm}$, was used to discriminate between the fluorescence and the excitation light (Figure S4). Overall, five different beams were used and the two-color point-scanning was performed as indicated (Figure 4).

With this approach we imaged HeLa cells expressing keratin19-rsEGFP2 together with vimentin-rsCherryRev1.4 with an overall pixel dwell-time of $\sim 6 \mathrm{~ms}$. Filaments formed by these proteins are strongly interweaved and in the confocal case the different filaments could often not be discerned, whereas the RESOLFT image allowed clear distinction (Figure $5 \mathrm{a}$ ). To demonstrate that rsCherryRev1.4 can also be imaged with other RSFPs than rsEGFP2, we expressed rsCherryRev1.4-MAP2 and vimentin-Dronpa-M159T in HeLa cells. In this case, Dronpa fluorescence was probed with $470 \mathrm{~nm}$, the off-switching light intensities were markedly reduced, and the overall pixel dwell time was slightly longer $(\sim 10 \mathrm{~ms})$ than with rsCherryRev1.4/ rsEGFP2 (Figure $5 b$, Table S3). Here the resolution value extracted from the labeled filaments in living cells was below $85 \mathrm{~nm}$ in both recording channels.

As for the single-color imaging with rsCherryRev1.4, no image processing was needed for the two-color imaging. The fluorescence cross-talk between both channels was negligible (Figure S8); the spatial overlap of both color channels was demonstrated by imaging cells co-expressing vimentin-rsCher-
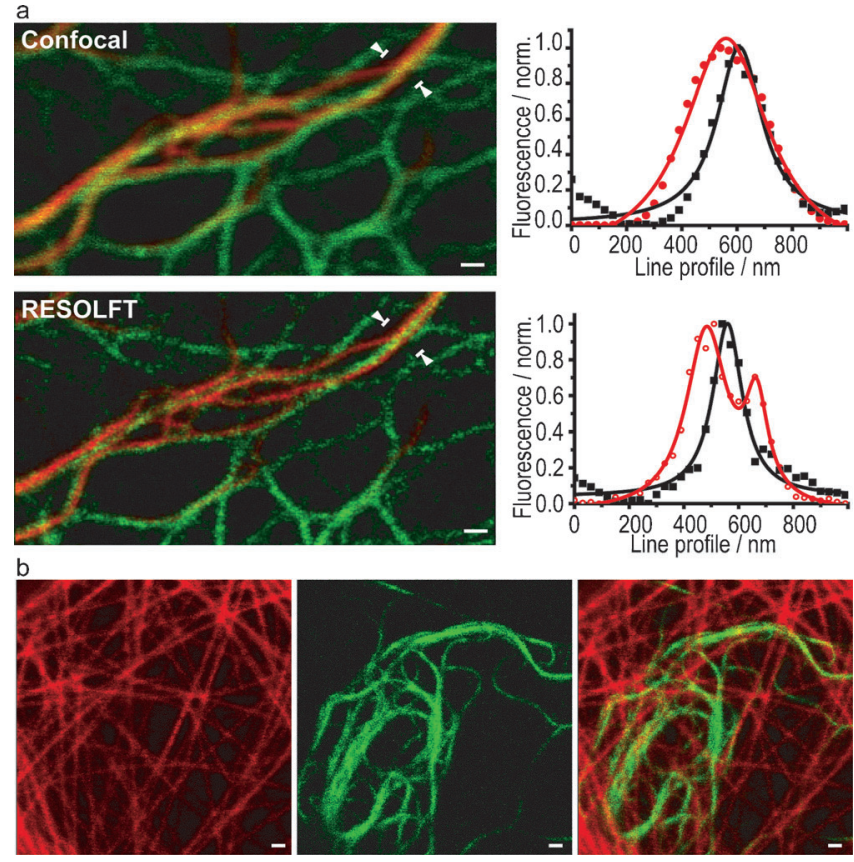

Figure 5. Confocal and RESOLFT imaging of living HeLa cells co-expressing green and red RSFP-fusion proteins. a) Confocal (top) and RESOLFT (bottom) images of a cell co-expressing vimentin-rsCherryRev1.4 (red) and keratin19rsEGFP2 (green). Graphs: Averaged line profiles over $100 \mathrm{~nm}$ across the region marked between the arrows. The line profiles of the data (dots) are shown with a Lorentzian fit (solid line) (rsCherryRev1.4: red, rsEGFP2: black) b) From left to right: RESOLFT images of rsCherryRev1.4-MAP2, vimentinDronpa-M159T, and overlay of both detection channels. Pixel step size: $30 \mathrm{~nm}$. Pixel dwell time: a) $5.8 \mathrm{~ms}$ and b) $10.2 \mathrm{~ms}$. Imaging times and intensities in Table S3. All images show raw data. Scale bars: $500 \mathrm{~nm}$.

ryRev1.4 together with vimentin-Dronpa-M159T (Figure S9). The superior discrimination of the green and red signals is due to the minimal overlap between the excitation and emission bands of the proteins as well as due to the sequential detection of the fluorescence. Since mathematical unmixing of the data of the two color channels is not required, only raw data are displayed.

\subsection{Parallelized Two-Color RESOLFT Nanoscopy}

Requiring comparatively low light intensities to overcome the diffraction barrier, RESOLFT nanoscopy lends itself for massive parallelization of the scanning procedure. This can be elegantly accomplished by the so-called incoherently crossed standing waves (ICS) pattern, an array of intensity zeros resulting from two orthogonally and incoherently superimposed standing light waves, as it has been recently demonstrated for green RSFPs. ${ }^{[13]}$ Therefore, we modified the reported parallelized RESOLFT setup ${ }^{[13]}$ so that we could explore the suitability of rsCherryRev1.4 for RESOLFT under massively parallelized scanning conditions. We created the ICS pattern of off-switching light at $592 \mathrm{~nm}$ and adapted the dichroic mirrors and fluorescence filters accordingly. For on-switching, light of $405 \mathrm{~nm}$ wavelength was used, while reading out was also performed by excitation at $592 \mathrm{~nm}$ wavelength. About $153 \times 153=23409$ 
minima resulted at the intersections of the line-shaped minima of the standing waves. Due to the inverse dependence of the resolution on the square-root of the intensity peak bordering the minima, for a resolution increase by $>2$-fold over that of a standard confocal microscope, these minima essentially behave like circular "donuts" providing isotropic resolution in the focal plane. ${ }^{[13]}$ The minima were simultaneously employed, covering a field of view of $50 \times 50 \mu \mathrm{m}$ so that the whole field was covered in $10 \times 10$ scanning steps. In this 24000 minima configuration, the power available from the laser for generating steep intensity minima was limited. Therefore, the switching cycle was 10 to 40 times longer than with the single-beam approach. However, the parallelization easily compensated for the longer switching times resulting in an overall recording time below 20 seconds for the $50 \times 50 \mu \mathrm{m}$-sized image. We imaged entire living HeLa cells expressing vimentin-rsCherryRev1.4, resolving small structures that were blurred in the corresponding wide-field image (Figure 6). We typically recorded five consecutive images (each recorded within $\sim 20 \mathrm{~s}$ ) with breaks of $10 \mathrm{~s}$ in between. After these five RESOLFT images the overall fluorescence intensity had decreased to $\sim 60 \%$ of the initial value, further demonstrating the photostability of rsCherryRev1.4 (Figure S10).

Finally, we extended the parallelized RESOLFT approach to two-color recording. To this end, HeLa cells expressing keratin19-rsCherryRev1.4 and vimentin-Dronpa-M159T were imaged in the parallelized mode (Figure 6). Both color channels were recorded consecutively. Due to axial chromatic aberrations in the imaging optical train (lens and achromat serving as a tube lens), refocusing was needed between the two recordings. The overall recording times were $<20$ seconds with rsCherryRev1.4 and $<5$ seconds with the green RSFP DronpaM159T for the $50 \times 50 \mu \mathrm{m}$-sized image enabling a resolution in both recording channels of $<110 \mathrm{~nm}$ in essentially raw data (Figure $6 \mathrm{f}$-j). As for two-color single-beam scanning imaging, the fluorescence cross-talk between both channels was negligible (Figure S11). Taken together, these results show that single and dual color RESOLFT nanoscopy utilizing red RSFPs can be parallelized by the ICS pattern, so that large fields of view can be superresolved within a few seconds.
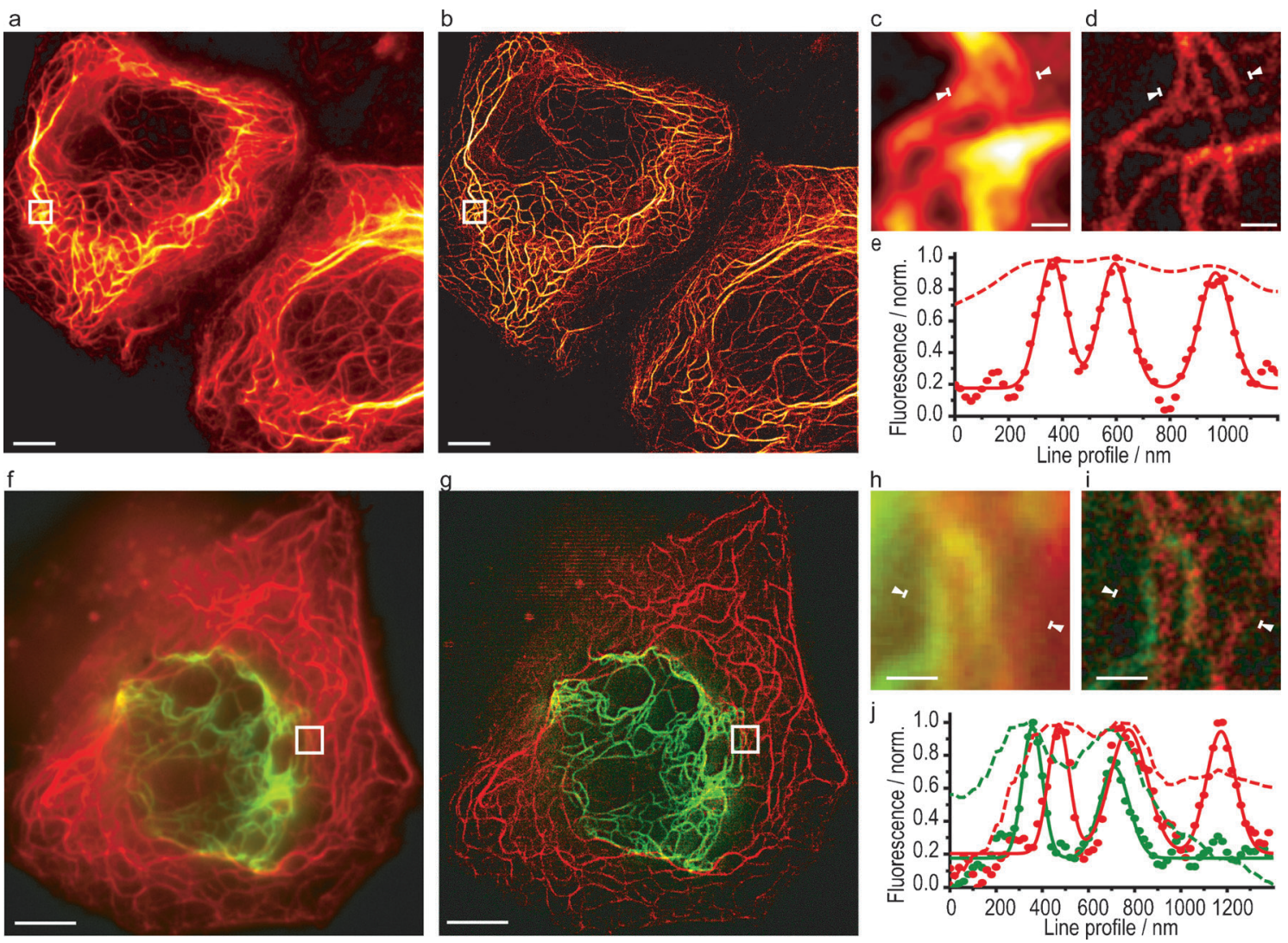

Figure 6. Parallelized RESOLFT nanoscopy. a)-e) Wide-field and RESOLFT images of live HeLa cells expressing keratin19-rsCherryRev1.4 recorded with a parallelized RESOLFT-setup. a) Wide-field and b) RESOLFT images with a field of view of $50 \times 50 \mu \mathrm{m} . \mathrm{c}$ ), d) Magnification of the regions marked in (a) and (b), respectively. e) Graph showing a line profile across the region between the arrows marked in (c) (dashed line) and (d) (dots). The RESOLFT data were fitted with three Gaussians (solid line). Each RESOLFT frame was acquired in $160 \mathrm{~ms}$ (on: $40 \mathrm{~ms}$, off: $100 \mathrm{~ms}$, read out : $20 \mathrm{~ms}$ ) and the total acquisition time including the frame rate of the CCD camera was about $18 \mathrm{~s}$. The RESOLFT image in (b) is displayed using a logarithmic color scale in order to highlight the smaller filaments. f)-j) Wide-field and RESOLFT two-color recording of live HeLa cells expressing keratin19-rsCherryRev1.4 (red) and vimentin-Dronpa-M159T (green). f) Wide-field and g) RESOLFT images with a field of view of $34 \times 36 \mu \mathrm{m}^{2}$. Each frame was acquired in $178 \mathrm{~ms}$ for the red channel and $37 \mathrm{~ms}$ for the green channel with a total imaging time of $\sim 4 \mathrm{~s}$ for the green channel and $\sim 19 \mathrm{~s}$ for the red channel. $\mathrm{h}$ ), $)$ Magnification of the regions marked in ( $\mathrm{f}$ ) and ( $\mathrm{g}$ ), respectively. j) Line profiles across the region between the arrows marked in (h) (dashed line) and (i) (dots). The solid lines represent Gaussian fits. The RESOLFT images were taken with 100 scanning steps. Scale bars: $5 \mu \mathrm{m}(\mathrm{a}, \mathrm{b}, \mathrm{f}, \mathrm{g})$ and $500 \mathrm{~nm}(\mathrm{c}, \mathrm{d}, \mathrm{h}, \mathrm{j})$. 


\section{Conclusion and Outlook}

In this study we demonstrated RESOLFT scanning nanoscopy with a red emitting reversibly switching fluorescent protein (RSFP), implemented both in a single-beam and in a highly parallelized manner. Co-expression of the red fluorescent RSFP rsCherryRev1.4 together with a green fluorescent RSFP enabled live-cell RESOLFT nanoscopy with two color channels; mathematical unmixing was not required for channel separation. While the resolution improvement gained by the RESOLFT method provides substantially finer image details than the corresponding diffraction-limited microscopy methods, the concept has not reached its full potential. A promising pathway for further improvements is protein engineering. While the development of rsCherryRev1.4 was an important factor for the implementation of RESOLFT in the red spectral regime, the protein still has limitations and is likely to be superseded by future versions of rsCherryRev or other red fluorescent RSFPs. rsCherryRev1.4 has a dimerization tendency and exhibits, similar to rsCherryRev, ${ }^{[17]}$ a complex photophysical behavior. Also, switching is not as fast as in rsEGFP2. ${ }^{[12 a]}$ While the design of switchable red fluorescent proteins proved to be notoriously challenging, red fluorescent proteins also offer a wide range of photochemical and photophysical variations, offering many possibilities for improvement by mutagenesis. ${ }^{[19]}$ Hence we expect the generation of further enhanced red fluorescent RSFPs to provide the pair of states and state transitions that ultimately yield higher resolution and imaging speed. Likewise, we anticipate that RESOLFT implementations that are perfectly matched to the state transition kinetics of the fluorophores in use will eventually enable high-speed, low light intensity dualcolor nanoscale imaging of living cells across large fields of view.

\section{Experimental Section}

\section{Single-Focused Beam Scanning RESOLFT and Confocal Imaging}

The point scanning confocal and RESOLFT imaging was carried out with a home-built stage-scanning RESOLFT microscope. A piezo stage (Melles-Griot, Carlsbad, USA) was used to scan over the sample. Up to six separate beam paths were used in the microscope for the reading-out and on- and off-switching of the red and green RSFPs. The on-switching of both proteins was performed with a regularly focused beam generated with a laser diode at $430 \mathrm{~nm}$ (LDH-D-C-430, $40 \mathrm{MHz}$, Picoquant, Berlin, Germany). Offswitching of the red RSFPs was performed with a donut- shaped beam generated by a continuous-wave fiber laser emitting at 592 nm (VFL-P-1000-592, CW, MPB Communications, Montreal, Canada). Fluorescence read-out of the red RSFPs was performed with a regularly focused beam generated by a diode-pumped solid-state laser at $561 \mathrm{~nm}$ (85-YCA-010, cw, Melles Griot, Carlsbad, USA). The donut-shaped off-switching beam and fluorescence read-out beam used for the green RSFPs were generated with the same laser diode at $488 \mathrm{~nm}$ (Sapphire-488-50, cW, Coherent, Santa Clara, USA). An additional beam for the green fluorescence excitation of $470 \mathrm{~nm}$ was generated with a laser diode at $470 \mathrm{~nm}$ (LDHP-C-470, $40 \mathrm{MHz}$, Picoquant, Berlin, Germany). The donut-shaped beams with a focal intensity distribution with a central minimum at the focus were obtained by using a vortex phase mask (VPP-1A, RPC Photonics, Rochester, NY). All laser beams were coupled into polarization maintaining single-mode fibers and, with exception of that of the $430 \mathrm{~nm}$ laser diode, were directed trough an acoustooptic modulator (AOM) (MQ180A0.25-VIS, AA Optoelectronics, Orsay, France) or acousto-optic tunable filter (AOTF) (AOTFnC-VIS, AA Optoelectronics) prior to the optical fiber. The acousto-optical devices were used for intensity control and triggering of the different beams. Intensity control of the $430 \mathrm{~nm}$ laser was performed with a liquid crystal modulator and a home-built electronic control card and triggering of the laser beam was carried out directly with the trigger input of the laser diode. All beams were co-aligned and coupled into a NA 1.4 oil-immersion lens (NA 1.4 HCX PL APO, 100x, Leica Microsystems, Wetzlar, Germany).

Pulse length and timing were defined by one or two pulse generators (PG) featuring 4 channels each (Model 9514, Quantum Composers, Bozeman, MT, USA). The first PG was used for the switching steps of the red RSFPs. It was triggered by a fast acquisition card (NiDAQmx, National Instruments Germany GmbH, Munich, Germany). The second PG was used for the switching of the green RSFPs and triggered directly by first PG. The respective outputs of the PGs were connected to the analog inputs of the AOMs or AOTFs, whereby the channel controlling the $430 \mathrm{~nm}$ beam was directly connected to the laser controller fast gating input (PDL-800D, Picoquant, Berlin, Germany). The fluorescence of the red RSFPs was filtered by a band-pass filter $(620 / 40 \mathrm{~nm})$ and the one of the green RSFPs with a $(525 / 50 \mathrm{~nm})$ band-pass filter. The fluorescence signals were detected on two different avalanche photo diodes (SPCMAQRH, PerkinElmer, Waltham, MA).

\section{Calculation of the Averaged Light Intensities in the Focal Plane}

All laser powers were measured at the entrance pupil of the objective lens and the transmission of the objective for the respective wavelength (between $75 \%$ and $85 \%$ for the wavelength used) was taken into account. The light intensity (in $\mathrm{kW} / \mathrm{cm}^{2}$ ) of the regularly focused (nearly Gaussian) beam in the focal plane was calculated according to Habuchi et al. ${ }^{[20]}$ and normalized to the donut area for the donut-shaped beams.

\section{Parallelized RESOLFT Imaging}

The parallelized microscope is largely based on a recently presented scheme. ${ }^{[13]}$ On-switching was performed with a $120 \mathrm{~mW}$ continuous wave $405 \mathrm{~nm}$ diode laser (LuxX 405-120, Omicron-laserage Laserprodukte, Rodgau, Germany). Off-switching and fluorescence read-out of rsCherryRev1.4 were carried out with a fiber laser at 592 nm (VFL-P-100-592, MPB communications, Montreal, Canada). Off-switching and reading-out of Dronpa-M159T was performed as described recently, ${ }_{1}^{[13]}$ using a $200 \mathrm{~mW}$ continuous wave $488 \mathrm{~nm}$ diode laser (LuxX 488-200, Omicron-laserage Laserprodukte, Rodgau, Germany). The on-switching beam ( $405 \mathrm{~nm}$ ) was modulated by directly applying TTL pulses to laser enable input. An acousto-optical modulator (MT110A1-VIS, AA optoelectronics, Orsay, France) was used for intensity control and triggering of the offswitching beam $(592 \mathrm{~nm})$. All beams were coupled into a singlemode polarization-maintaining PC/APC fiber (P5-488PM-FC-2, Thorlabs, Newton, USA). All laser beams exiting the fiber were collimated with an achromatic doublet $(f=35 \mathrm{~mm})$ and directed through a half wave plate and a polarizing beamsplitter (PBS). After the PBS the beams were split into two paths and directed through custom- 
made phase diffraction gratings, each with $437 \mathrm{~nm}$ high $\mathrm{SiO}_{2}$ lines with a $36 \mu \mathrm{m}$ period (Laser Laboratorium, Göttingen, Germany). The gratings' line-orientation was adjusted to be parallel to the polarization of the incoming light with a precision rotation mount. Using a lens of $100 \mathrm{~mm}$ focal length, the diffracted light was focused to a secondary pupil and all of the diffraction orders except +1 and -1 were blocked. These orders were relayed by a pair of lenses $(f=200 \mathrm{~mm}$ ) to four spots in the back focal plane (BFP) of a $100 \times$, NA 1.45 objective lens (Olympus, Shinjuku, Japan), where they recombined and created a regular sinusoidal pattern in two orientations. The fluorescence was collected by the same objective lens and separated by a custom multi-color dichroic mirror (59022BS-UV), filtered using a fluorescence filter (BrightLine HC641/ $75)$, directed through a tube lens $(f=300 \mathrm{~mm})$ and imaged onto an electron-multiplying CCD camera (iXon 897, Andor Technology, Belfast, UK), which was cooled to $-80^{\circ} \mathrm{C}$. The focal length of the tube lens was chosen in such a way that the $16 \mu \mathrm{m}$ pixels on the camera project to $96 \mathrm{~nm}$ pixels in sample space. Scanning of the sample was realized using a three axes piezo stage (NanoMax, Thorlabs, Newton, USA). Since the fluorescence switching-off and read-out was done using the same pattern, the sample was translated half a period of the pattern between these two steps.

\section{Screening and Low-Intensity Switching Experiments}

The screening of mutant libraries and the low intensity photoswitching experiments were performed on $E$. coli colonies expressing the respective protein using a modified computer-controlled fluorescence microscope (Leica) equipped with a $50 \times$ NA 0.5 air objective lens essentially as described previously. ${ }^{[11 \mathrm{~d}]}$ Two $100 \mathrm{~W} \mathrm{Hg}$ lamps delivering yellow light $(577 \pm 5 \mathrm{~nm}$ excitation filter, $\left.\sim 40 \mathrm{~W} \mathrm{~m}^{-2}\right)$ or blue light $\left(450 \pm 20 \mathrm{~nm}\right.$ excitation filter, $\left.\sim 10 \mathrm{~W} \mathrm{~cm}^{-2}\right)$ were used for excitation and switching. The fluorescence was detected through the same objective lens and recorded by a photomultiplier tube (HR9306-0, Hamamatsu, Hamamatsu City, Japan) using a $600 \mathrm{~nm}$ long-pass detection filter.

\section{Absorption and Emission Spectra}

Absorption and emission spectra were recorded on a Varian Cary 4000 UV/VIS spectrophotometer and a Varian Cary Eclipse fluorescence spectrometer (Agilent, Santa Clara, USA), respectively. Before measuring the emission spectrum, the protein solution was switched into the on-state with light of $450 / 40 \mathrm{~nm}$.

\section{Wide-field Measurements}

For wide-field imaging, an epifluorescence microscope (DM6000, Leica Microsystems, Wetzlar, Germany) equipped with a $63 \times$ NA 1.2 water immersion objective lens and a camera (DFC350 FX, Leica Microsystems, Wetzlar, Germany) were used. The fluorescence images were recorded with blue and green excitation light (420/30 and $570 / 20 \mathrm{~nm}$ ). For fluorescence detection, a $590 \mathrm{~nm}$ dichroic mirror and a $640 / 40 \mathrm{~nm}$ detection filter were used. The duration of the image acquisition was adjusted to the brightness of every sample. For quantification of brightness of mammalian cells, a $20 \times$ NA 0.5 air objective lens and the same imaging parameters were used for all images taken. The average intensity of single rsCherryRev or rsCherryRev1.4 fusion protein expressing cells was quantified using ImageJ.

\section{Protein Expression and Mutagenesis}

For protein expression and mutagenesis, the respective coding sequences were PCR amplified, digested and inserted into the BamHI and HindIII restriction sites of the high copy expression vector pQE31 (Qiagen, Venlo, Netherlands). Site directed mutagenesis was performed using the QuikChange Site Directed Mutagenesis Kit (Stratagene, Santa Clara, USA) and random mutagenesis was performed as described previously. ${ }^{[11]}$

\section{Protein Purification}

Proteins were expressed in the Escherichia coli strain BL21-CP-RIL and purified by Ni-NTA affinity chromatography, followed by a gelfiltration step on a Superdex 200 column (Amersham Biosciences) using a Tris-buffer (100 mm Tris-HCl, 150 m M NaCl, pH 7.5).

\section{Chromophore Maturation}

To determine the chromophore maturation time of rsCherryRev, TOP10 E. coli cells were transformed with pBAD-rsCherryRev and pBAD-rsCherryRev1.4. Overnight cultures of these Top10 cells were used to inoculate $100 \mathrm{~mL}$ of LB-medium supplemented with $100 \mu \mathrm{g} \mathrm{mL} \mathrm{L}^{-1}$ ampicillin and grown at $37^{\circ} \mathrm{C}$ to an $\mathrm{OD}_{600}$ of 0.5 . After addition of $0,2 \%$ arabinose, the cultures were cultivated under low-oxygen conditions for $3 \mathrm{~h}$. Subsequently, the cells were pelleted and disrupted. The proteins were immediately purified using His SpinTrap columns (GE Healthcare) within $30 \mathrm{~min}$ and kept strictly at $4{ }^{\circ} \mathrm{C}$. The purified proteins were diluted in $20 \mathrm{~mm}$ $\mathrm{NaH}_{2} \mathrm{PO}_{4}, 500 \mathrm{~mm} \mathrm{NaCl}, 30 \mathrm{~mm}$ imidazol, pH 7.5. Finally, the fluorescence of the protein solution was measured at different time points using the described microscopic switching setup using excitation filters with $577 / 10$ and $450 / 40 \mathrm{~nm}$, while the protein solution was incubated at $37^{\circ} \mathrm{C}$.

\section{Semi-Native Polyacrylamide Gel Electrophoresis}

For semi-native polyacrylamide gel electrophoresis (PAGE), $20 \mu \mathrm{g}$ of each protein were taken up in a concentrated sucrose solution to a final concentration of $10 \%(\mathrm{w} / \mathrm{v})$ sucrose and loaded on a $12.5 \%$ polyacrylamide gel containing $0.1 \%$ sodiumdodecyl sulphate. Images were taken with a custom built gel documentation system as described previously. ${ }^{[11 c]}$

\section{Expression in Mammalian Cells}

To target rsCherryRev and rsCherryRev1.4 to the lumen of the endoplasmic reticulum (ER), the coding sequences of the proteins were amplified by PCR. The PCR fragments were digested with Sall and Notl and inserted into the vector $\mathrm{pEF} / \mathrm{myc} / \mathrm{ER}$ (Invitrogen, Carlsbad, USA). For the generation of the $\alpha$-tubulin fusion construct, the GFP sequence was cut out of the vector pEGFP-Tub (Clontech, Mountain View, USA) using Nhel and Bg/ll and replaced by the rsCherryRev1.4 sequence. To generate the microtubule-associated protein 2 (MAP2) fusion construct, the tubulin sequence was swapped with the MAP2 (obtained from pDONR223-MAP2) sequence using the $\mathrm{Xhol}$ and $\mathrm{BamHI}$ restriction sites of prsCherryRev1.4. For the creation of fusion constructs of rsCherryRev1.4, rsEGFP2 or Dronpa-M159T with caveolin, keratin19 or vimentin, the respective sequences were amplified by PCR. The PCR fragments were cloned into the gateway destination vector pMD-tdEosFP-N using the restriction sites Agel and Afll, thereby replacing the tdEosFP coding sequence. The final plasmids pMD-Vim-rsCherryRev1.4, pMD-Vim-Dronpa-M159T, pMD-Ker19- rsCherryRev1.4, pMDKer19-rsEGFP2 and pMD-Cav1- rsCherryRev1.4 were constructed by 
gateway vector conversion (Invitrogen, Carlsbad, USA) using the donor vectors pDONR223-Vim, pDONR223-Krt19 and pDONR223Cav1. ${ }^{[21]}$

HeLa and PtK2 cells were cultured at $37^{\circ} \mathrm{C}$ with $5 \% \mathrm{CO}_{2}$ in DMEM (Invitrogen, Carlsbad, USA) containing 10\% FCS (PAA Laboratories, Cölbe, Germany), $1 \mathrm{~mm}$ pyruvate (Sigma, St-Louis, USA), $100 \mu \mathrm{gL}^{-1}$ streptomycin and $100 \mu \mathrm{g} \mathrm{mL}^{-1}$ penicillin (Biochrom, Berlin, Germany). Cells were seeded on coverslips in six-well plates and transiently transfected with the plasmids using $4 \mu \mathrm{g}$ DNA ( $2 \mu \mathrm{g}$ DNA each construct for double transfections) and Turbofect (Thermo Scientific, Waltham, USA) the following day according to the manufacturer's instructions. 24 to $48 \mathrm{~h}$ after transfection, the cells were transferred in phenol-red free DMEM (Invitrogen, Carlsbad, USA), mounted on concavity slides and sealed with twinsil (Picodent, Wipperfürth, Germany). Live-cell imaging was performed at room temperature.

\section{Acknowledgements}

We thank J. Jethwa for critical reading of the manuscript and T. Gilat and S. Löbermann for excellent technical assistance. Part of the work was supported by the Deutsche Forschungsgemeinschaft through the DFG-Cluster of Excellence "Nanoscale Microscopy and Molecular Physiology of the Brain" (to SJ and SWH).

\section{Keywords: fluorescent proteins . nanoscopy photoswitching $\cdot$ RESOLFT $\cdot$ superresolution imaging}

[1] E. Abbe, Arch. Mikrosk. Anat. 1873, 9, 413-468.

[2] a) M. Dyba, S. Jakobs, S. W. Hell, Nat. Biotechnol. 2003, 21, 1303-1304; b) T. A. Klar, S. Jakobs, M. Dyba, A. Egner, S. W. Hell, Proc. Natl. Acad. Sci. USA 2000, 97, 8206-8210; c) S. W. Hell, Nat. Biotechnol. 2003, 21, $1347-$ 1355.

[3] a) S. W. Hell, Nat. Methods 2009, 6, 24-32; b) S. W. Hell in Single-Molecule Spectroscopy in Chemistry (Ed.: A. Gräslund, R. Rigler, J. Widengren), Springer, Berlin, 2009, pp. 365-398.

[4] E. Betzig, G. H. Patterson, R. Sougrat, O. W. Lindwasser, S. Olenych, J. S. Bonifacino, M. W. Davidson, J. Lippincott-Schwartz, H. F. Hess, Science 2006, 313, 1642-1645.

[5] M. J. Rust, M. Bates, X. W. Zhuang, Nat. Methods 2006, 3, 793-795.

[6] J. Fölling, M. Bossi, H. Bock, R. Medda, C. A. Wurm, B. Hein, S. Jakobs, C. Eggeling, S. W. Hell, Nat. Methods 2008, 5, 943-945.
[7] M. Heilemann, S. van de Linde, M. Schuttpelz, R. Kasper, B. Seefeldt, A. Mukherjee, P. Tinnefeld, M. Sauer, Angew. Chem. 2008, 120, 6266-6271; Angew. Chem. Int. Ed. 2008, 47, 6172-6176.

[8] J. S. Biteen, M. A. Thompson, N. K. Tselentis, G. R. Bowman, L. Shapiro, W. E. Moerner, Nat. Methods 2008, 5, 947-949.

[9] S. W. Hell, J. Wichmann, Opt. Lett. 1994, 19, 780-782.

[10] a) M. G. L. Gustafsson, Proc. Natl. Acad. Sci. USA 2005, 102, 13081 13086 ; b) R. Heintzmann, T. M. Jovin, C. Cremer, J. Opt. Soc. Am. A 2002, 19, $1599-1609$.

[11] a) M. Hofmann, C. Eggeling, S. Jakobs, S. W. Hell, Proc. Natl. Acad. Sci. USA 2005, 102, 17565-17569; b) S. W. Hell, S. Jakobs, L. Kastrup, Appl. Phys. A 2003, 77, 859-860; c) T. Grotjohann, I. Testa, M. Leutenegger, H. Bock, N. T. Urban, F. Lavoie-Cardinal, K. I. Willig, C. Eggeling, S. Jakobs, S. W. Hell, Nature 2011, 478, 204-208; d) T. Brakemann, A. C. Stiel, G. Weber, M. Andresen, I. Testa, T. Grotjohann, M. Leutenegger, U. Plessmann, H. Urlaub, C. Eggeling, M. C. Wahl, S. W. Hell, S. Jakobs, Nat. Biotechnol. 2011, 29, 942-947.

[12] a) T. Grotjohann, I. Testa, M. Reuss, T. Brakemann, C. Eggeling, S. W. Hell, S. Jakobs, eLife 2012, 1, e00248; b) S. Berning, K. I. Willig, H. Steffens, P. Dibaj, S. W. Hell, Science 2012, 335, 551; c) V. Westphal, S. O. Rizzoli, M. A. Lauterbach, D. Kamin, R. Jahn, S. W. Hell, Science 2008, 320, 246 249.

[13] A. Chmyrov, J. Keller, T. Grotjohann, M. Ratz, E. d'Este, S. Jakobs, C. Eggeling, S. W. Hell, Nat. Methods 2013, 10, 737-740.

[14] I. Testa, N. T. Urban, S. Jakobs, C. Eggeling, K. I. Willig, S. W. Hell, Neuron 2012, 75, $992-1000$.

[15] A. C. Stiel, S. Trowitzsch, G. Weber, M. Andresen, C. Eggeling, S. W. Hell, S. Jakobs, M. C. Wahl, Biochem. J. 2007, 402, 35-42.

[16] W. E. Moerner, R. M. Dickson, A. B. Cubitt, R. Y. Tsien, Nature 1997, 388, $355-358$

[17] A. C. Stiel, M. Andresen, H. Bock, M. Hilbert, J. Schilde, A. Schonle, C. Eggeling, A. Egner, S.W. Hell, S. Jakobs, Biophys. J. 2008, 95, 29892997.

[18] F. V. Subach, L. Zhang, T. W. J. Gadella, N. G. Gurskaya, K. A. Lukyanov, V. V. Verkhusha, Chem. Biol. 2010, 17, 745-755.

[19] F. V. Subach, V. V. Verkhusha, Chem. Rev. 2012, 112, 4308-4327.

[20] S. Habuchi, P. Dedecker, J. I. Hotta, C. Flors, R. Ando, H. Mizuno, A. Miyawaki, J. Hofkens, Photochem. Photobiol. Sci. 2006, 5, 567-576.

[21] P. Lamesch, N. Li, S. Milstein, C. Y. Fan, T. Hao, G. Szabo, Z. J. Hu, K. Venkatesan, G. Bethel, P. Martin, J. Rogers, S. Lawlor, S. McLaren, A. Dricot, H. Borick, M. E. Cusick, J. Vandenhaute, I. Dunham, D. E. Hill, M. Vidal, Genomics 2007, 89, 307-315.

Received: November 4, 2013

Published online on $\mathbf{\square}$ II, 2014 


\section{ARTICLES}

f. Lavoie-Cardinal, N. A. Jensen, V. Westphal, A. C. Stiel, A. Chmyrov,

J. Bierwagen, I. Testa, S. Jakobs, *

S. W. Hell*

$\square-\square$

Two-Color RESOLFT Nanoscopy with Green and Red Fluorescent Photochromic Proteins

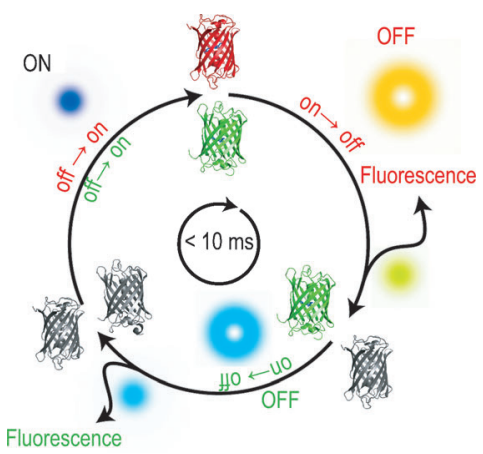

Look lively! Reversible saturable optical fluorescence transitions (RESOLFT) nanoscopy with the new red fluorescent photochromic protein rsCherryRev1.4 enables single- and two-color live cell subdiffraction resolution imaging with a single-point scanning and a massively parallelized approach. 